\title{
A study of correlation between IceCube neutrino events and Ultra high energy cosmic rays
}

\author{
Reetanjali Moharana* \\ Department of Physics, University of Johannesburg, Auckland Park 2009, South Africa \\ E-mail: reetanjalimeuj.ac.za \\ Soebur Razzaque \\ Department of Physics, University of Johannesburg, Auckland Park 2009, South Africa \\ E-mail: srazzaque@uj.ac.za
}

\begin{abstract}
Sources for cosmic neutrino events detected by the IceCube Neutrino Observatory with energy above $30 \mathrm{TeV}$ are hard to find due to their poor angular resolutions. Ultrahigh-energy cosmic rays (UHECRs) are detected with better angular resolutions above $100 \mathrm{EeV}$ energies and can be used to check if the same astrophysical sources are responsible for producing both neutrinos and UHECRs. We test the correlation of UHECR data of the Pierre Auger Observatory, Telescope Array and past cosmic-ray experiments with IceCube neutrino events using statistical methods which emphasize invariant quantities. We find that the arrival directions of the $3 y r$ IceCube detected cosmic neutrinos are correlated with $\geq 100 \mathrm{EeV}$ UHECR arrival directions at confidence level $\sim 90 \%$. In our recent approach we have tried to find a correlation of the $4 \mathrm{yr}$ IceCube detected High-Energy Starting Events (HESE) neutrino events with UHECRs from PAO and TA. We found more than $3 \sigma$ correlation for PAO only and TA+PAO UHECRs events $>100 \mathrm{EeV}$.
\end{abstract}

$3 r d$ Annual Conference on High Energy Astrophysics in Southern Africa-HEASA2015,

18-20 June 2015

University of Johannesburg, Auckland Park, South Africa

\footnotetext{
*Speaker.
} 


\section{Introduction}

IceCube Neutrino Observatory at South pole is the world's largest neutrino detector built in 1 $\mathrm{km}^{3}$ volume ice, and has observed neutrino events within energy range $\sim 20 \mathrm{TeV}-2.3 \mathrm{PeV}$ range in last 4 years [1,2]. 37 neutrino events were detected in the first $3 \mathrm{yr}$ of IceCube observation. Shower events, most likely due to $v_{e}$ or $v_{\tau}$ charge current $v N$ interactions and also due to neutral current $v N$ interactions of all flavors, dominate the event list (28 including 3 events with 1-2 PeV energy) while track events, most likely due to $v_{\mu}$ charge current $v N$ interactions, constitute the rest out of the $3 \mathrm{yr}$ data. Among these 37 events about 15 could be due to atmospheric neutrinos $\left(6.6_{-1.6}^{+5.9}\right)$ and muon (8.4 \pm 4.2$)$ backgrounds. A background-only origin of all 37 events has been rejected at 5.7- $\sigma$ level [1]. A similar observation has also seen for 4 yr IceCube data set [2].

UHECRs are the other high energy particles, mostly protons or heavy nuclei, detected from extragalactic origin. Pierre Auger Observatory (PAO) [3] and the Telescope Array (TA) [4], two of the world's largest operating cosmic-ray detectors, have recently released UHECR data collected over more than 10-year and 5-year periods, respectively. Together they have detected 16 events (6 by PAO [3] and 10 by TA [4]) with energies $\gtrsim 100 \mathrm{EeV}$. The total publicly available $\gtrsim 100 \mathrm{EeV}$ events including past experiments is 33 . Lower energy cosmic rays may get deflected from the arrival directions by the Galactic and intergalactic magnetic fields. However at $\gtrsim 60 \mathrm{EeV}$ energies the arrival directions of UHECRs tend to be much better correlated with their source directions and astronomy with charged particles could be realized [5]. The astrophysical sources of UHECRs with energy $\gtrsim 40 \mathrm{EeV}$ need to be located within the so-called GZK volume [6, 7] in order to avoid serious attenuation of flux from them due to interactions of UHECRs with photons from cosmic microwave background (CMB). The astrophysical sources of neutrinos, on the other hand, can be located at large distances and still be detected provided their luminosity is sufficiently high.

We explore here a possibility that both UHECRs and IceCube cosmic neutrino events are produced by the same astrophysical sources within the GZK volume. Since widely accepted Fermi acceleration mechanism of cosmic rays at the sources take place over a large energy range, it is natural that the same sources produce $\gtrsim 1 \mathrm{PeV}$ cosmic rays, required to produce cosmic neutrinos observed with energies down to $\sim 30 \mathrm{TeV}$, and UHECRs. We studied the correlation of UHECRs within the angular errors IceCube neutrino events . Existence of such a correlation can provide clues to the origin of both cosmic neutrinos and UHECRs.

In order to pursue our study we used two sets of the IceCube data and two different analysis methods. 1) We employ invariant statistical method [8], independent of coordinate systems, in order to study angular correlation between the first $3 \mathrm{yr}$ cosmic neutrinos of IceCube and the UHECRs. 2) Cross correlation method [9] is used to study the correlation of the 4 yr neutrinos within energy $20 \mathrm{TeV}$ to $2.3 \mathrm{PeV}$ and the UHECRs above $>100 \mathrm{EeV}$.

\section{IceCube neutrino events and UHECR data}

In the first analysis we used the 35 IceCube neutrino events, collected over the first 988 days in the $\sim 30 \mathrm{TeV}-2 \mathrm{PeV}$ energy range, from ref. [1] to study angular correlation with UHECRs. Two track events (event numbers 28 and 32) are coincident hits in the IceTop surface array and are almost certainly a pair of atmospheric muon background events [1]. Therefore we excluded them 
from our analysis. The angular resolutions of the track events are $\lesssim 1.4^{\circ}$ and those of the shower events vary between $6.6^{\circ}$ and $46.3^{\circ}$.

The second analysis include 52 IceCube events collected over 1347 days excluding the 2 muon tracks mentioned above. Out of these 54 events detected within the $4 \mathrm{yrs}$ include an estimated atmospheric neutrino background $9.0_{-2.2}^{+8.0}$ and atmospheric muons $12.6 \pm 5.1$ [2]. So the number of events seen is not from atmospheric origin with a confidence of $6.5 \sigma$ [2]. These events have similar angular resolution as the $3 \mathrm{yr}$ neutrino events.

The detail of UHECR events used are as follow. The PAO and TA collaborations have published data with energies above $52 \mathrm{EeV}$ (231 events) [3] and $57 \mathrm{EeV}$ (72 events) [4], respectively. Note that the PAO and TA are located in the southern and northern hemisphere, respectively, covering a declination range of $-90^{\circ} \leq \mathrm{Dec} \leq 45^{\circ}$ [3] and $-10^{\circ} \leq \mathrm{Dec} \leq 90^{\circ}$ [4]. The angular resolutions for the PAO events with energy $>10 \mathrm{EeV}$ is $<0.9^{\circ}$ [10] while for the TA events with energy $>57 \mathrm{EeV}$ it is between $1.0^{\circ}$ and $1.7^{\circ}$ [4]. AGASA experiment has published data above 40 $\mathrm{EeV}$ (40 events) covering a declination range $-10^{\circ} \leq \mathrm{Dec} \leq 90^{\circ}$ and with angular resolution $<2^{\circ}$ [11]. Only $\geq 100 \mathrm{EeV}$ data are available from the other past experiments: Haverah Park [12, 13], Yakutsk [12], Volcano Ranch [12] and Fly's eye [12]. We could not include 13 events with energy $>56 \mathrm{EeV}$ from the HiRes experiment as the energies of the individual events are not available [14].

The publicly available earlier UHECR data includes 6 events from Haverah Park, 1 event from Yakutsk, 8 events from AGASA, 1 event from Volcano Ranch, the highest-energy (320 EeV) event from Fly's eye above energy $100 \mathrm{EeV}$. We use these along with PAO and TA data separately to study correlation with cosmic neutrino events.

\section{Statistical method and data analyses}

To study correlation between cosmic neutrinos and UHECRs, we map the event directions into unit vectors on a sphere as $\hat{x}=(\sin \theta \cos \phi, \sin \theta \sin \phi, \cos \theta)^{T}$, where $\phi=R A$ and $\theta=\pi / 2-D e c$. The angle between the two vectors $\gamma=\cos ^{-1}\left(\hat{x}_{\text {neutrino }} \cdot \hat{x}_{\text {UHECR }}\right)$, is an invariant measure of the angular correlation between the neutrino and UHECR arrival directions [8]. In our first method of analysis following we use statistic made from invariant $\gamma$ for each neutrino direction $\hat{x}_{i}$ and UHECR direction $\hat{x}_{j}$ pair as

$$
\delta \chi_{i}^{2}=\min _{j}\left(\gamma_{i j}^{2} / \delta \theta_{i}^{2}\right),
$$

which is minimized for all $j$. Here $\delta \theta_{i}$ is the angular resolution of the detected IceCube neutrino events. We use the exact resolutions reported by the IceCube collaboration for each event [1]. A value $\delta \chi_{i}^{2} \leq 1$ is considered a "good match" between the $i$-th neutrino and an UHECR arrival directions. The distribution with observed data giving a number of "hits" or $N_{\text {hits }}$ with $\delta \chi^{2} \leq 1$ therefore forms a basis to claim correlation. Note that in case more than one UHECR directions are within the error circle of a neutrino event, the $\delta \chi^{2}$ value for UHECR closest to the neutrino direction is chosen in this method. We estimate the significance of any correlation in data by comparing $N_{\text {hits }}$ with corresponding number from null distributions. We calculate statistical significance of correlation in real data or $p$-value (chance probability) using frequentists' approach. We count the number of times we get a random data set that gives equal or more hits than the $N_{\text {hits }}$ in real data within $\delta \chi^{2} \leq 1$ bin. Dividing this number with the total number of random data sets generated $\left(10^{5}\right)$ gives 
us the $p$-value. We cross-check this $p$-value by calculating the Poisson probability of obtaining $N_{\text {hits }}$ within $\delta \chi^{2} \leq 1$ bin given the corresponding average hits expected from the null distribution.

The second method named as the cross correlation is based on the number of neutrino and UHECR pairs observed within a given angular region. We divided $\delta \theta$ into $M=10$ concentric rings, each with angular width $\delta \theta / M$. We count the number of pairs $n_{j}^{\text {data }}$ in a given annular $\operatorname{ring}(j)$. To get the confidence of correlation, we generate a large number sets of Monte Carlo UHECR event distributions by randomizing their positions and then counting the number of pairs in a given annular ring. The average number of pairs $\left(\bar{n}_{j}^{m c}\right)$ and the variance $\left(\sigma_{j}\right)$ in each ring help in calculating the relative excess $\left(n_{j}^{\text {data }}-\bar{n}_{j}^{m c}\right) / \sigma_{j}$ in a given ring. A larger positive value for the relative excess corresponds to higher significance that the neutrinos and UHECRs are correlated. A negative value for the relative excess, on the other hand, represents anti-correlation between them. The relative excess represents the Gaussian distribution for the correlation, $\sigma$, and p-value is calculated corresponding to this $\sigma$.

We construct two null distributions, in one case we randomize only the $R A$ of the UHECRs, keeping their Dec the same as in data; and in the second case we also randomize Dec according to the zenith-angle depended sky exposure of the UHECR experiments along with the RAs [19], affecting the declination distributions of UHECR data. We call these two null distributions as the semi-isotropic null and exposure-corrected null, respectively. The semi-isotropic null is a quick-way to check significance while the exposure-corrected null is accurate when information on particular experiments are available. We find that the two null distributions are in good agreement with each other in most cases.

\section{Results}

\subsection{Correlations between neutrinos and UHECRs}

We apply our first statistical method separately to the PAO and TA data, to a combination of both and to all available UHECR data above $100 \mathrm{EeV}$ from all experiments while for the second method we have used separately to the PAO and TA data, to a combination of the both. Figure 1 shows the result of correlation study in case of first method of analysis. The counts in the $0 \leq$ $\delta \chi^{2} \leq 1$ bins shown with the blue filled histograms correspond to the number of correlated neutrino events with the UHECRs. Counts in other bins are due to distant pairs of the neutrino events and UHECRs and are uninteresting for us. The counts with the red (green), open histogram in the same bins correspond to the expected number of correlated neutrino events from the semi-isotropic null (exposure-corrected null). Both null distributions give similar results.

Figure 1 also shows $p$-values or the probability of finding the correlated events $\left(0 \leq \delta \chi^{2} \leq 1\right)$ in observed data as a fluctuation of the randomly distributed UHECRs in the sky. The probability $1-p \approx 90 \%$ is the confidence level (CL) that the IceCube neutrino events and all available UHECR data with energy $\geq 100 \mathrm{EeV}$ are correlated. A correlation with similar CL exists between the neutrino and PAO-only data sets and between the neutrino and TA-only data sets. The Poisson probability of obtaining $N_{\text {hits }}=7$ in PAO data when 4 are expected from the semi-isotropic null distribution and $N_{\text {hits }}=6$ in TA data with 3.8 expected from the same null distribution are $\approx 0.06$, in very good agreement with our $p$-values. Similarly, for the combined data set of all UHECRs 

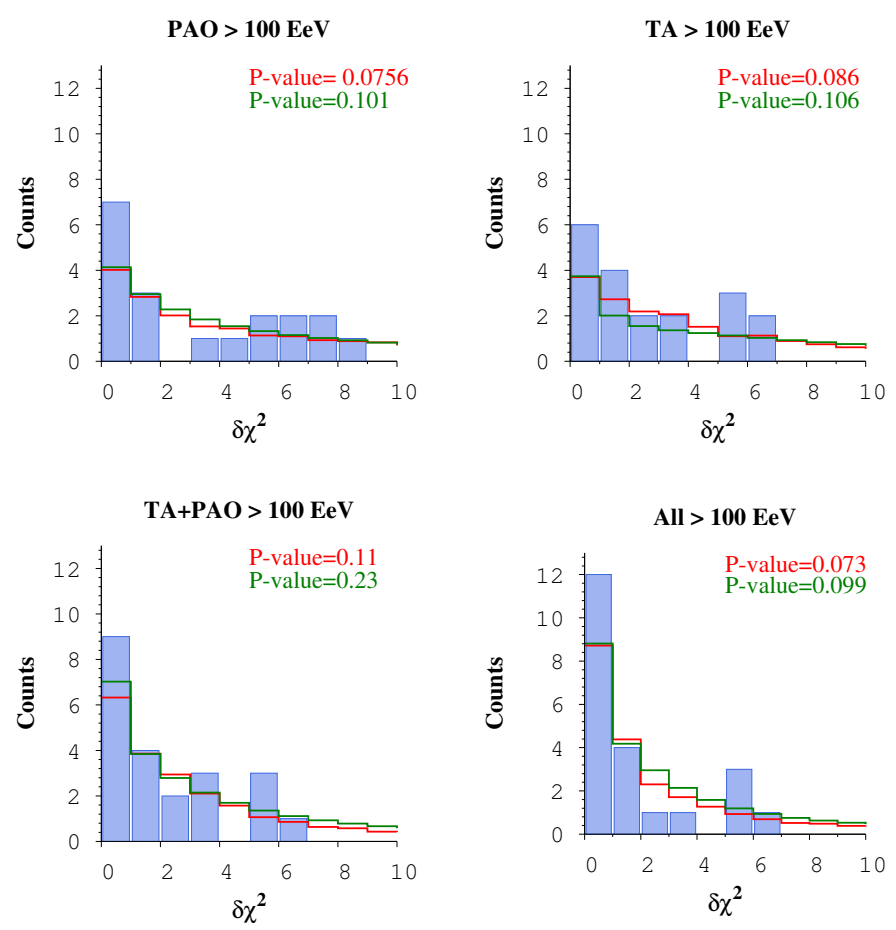

Figure 1: Distributions of $\delta \chi^{2}$ found in the observed data (blue, filled histograms) and in the simulated data corresponding to the semi-isotropic null (red, open histograms) and the exposure-corrected null (green, open histograms). The histograms have been truncated at $\delta \chi^{2}=10$ for better display. The significance ( $p$-values) have been calculated for the $0 \leq \delta \chi^{2} \leq 1$ bins.

$>100 \mathrm{EeV}, N_{\text {hits }}=12$ when expected value is 8.8 corresponds to a Poisson probability of $\approx 0.07$, again in very good agreement with our $p$-value.

We remind the readers that the counts in the $\delta \chi^{2}$ distributions with TA+PAO data is not the algebraic sum of the counts in the distributions with TA and PAO data separately. This is because our $\delta \chi^{2}$ statistic choose the nearest UHECR data point even if more than one are present within the error circle of a neutrino event. The same is true for distribution with all UHECRs. Note that we list all UHECRs giving $\delta \chi^{2} \leq 1$ in the table. There are 7 UHECRs which are correlated with 2 or more neutrino events. None of the correlated neutrinos are PeV neutrino events.

Figure 2 shows the result for second method of analysis. The PAO only, TA only and TA+PAO relative excess are shown with pink, blue and black color box respectively. We have used only isotropic null distribution for doing this analysis and generated $10^{5}$ Monte Carlo UHECRs. In case of PAO only we get the maximum relative excess, 3.6 (3.6 $\sigma$ of correlation) within the $0-0.1 \delta \theta$ bin, and the corresponding $p$-value $2.6 \times 10^{-4}$. However in case of TA-only the significance decreased. The maximum relative excess is 1.5 (1.5 $\sigma$ of correlation) within the $0.4-0.5 \delta \theta$ bin, and the corresponding $p$-value 0.12 . In case of TA+PAO the significance is less than PAO only. The maximum relative excess is 2.46 (2.46 $\sigma$ of correlation) within the $0-0.1 \delta \theta$ bin, and the corresponding $p$-value 

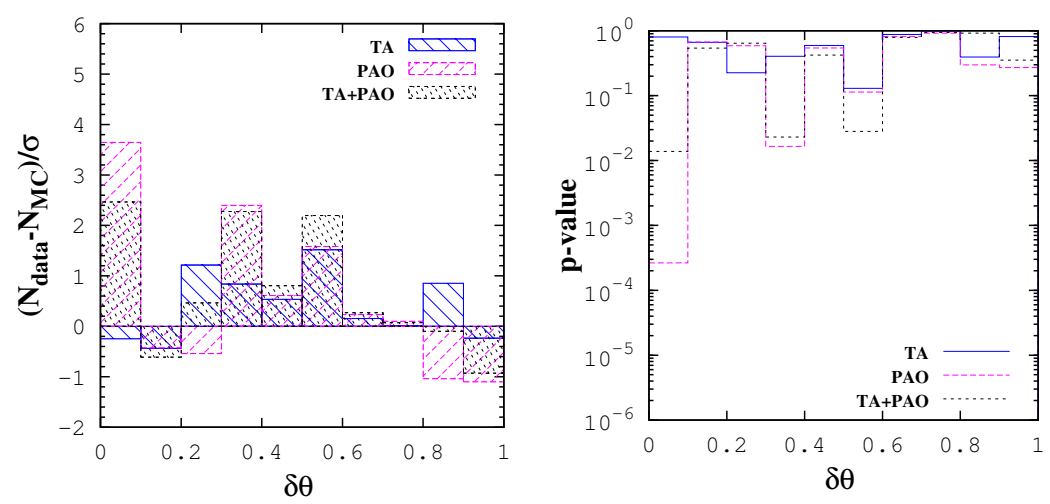

Figure 2: The left panel of the figure shows the relative excess of data over isotropic distribution for UHECRs by PAO only (pink color box), TA only (blue box) and TA+PAO (black box) in 10 small concentric rings of $\delta \theta$. And the $\mathrm{p}$-value for the corresponding rings is shown in the right panel with same color lines as left panel with 4 yr data of IceCube.

$1.4 \times 10^{-2}$

\subsection{Energy calibrated events}

Energy calibration among different UHECR experiments is a widely-debated issue. If UHECR flux is uniform over the whole sky and each experiment measures the same primary particles' energy then the number of UHECR events should be proportional to the exposures of the experiments. Therefore it is difficult, in particular, to reconcile the $10 \mathrm{TA}$ events at $>100 \mathrm{EeV}$ compared to the 6 from PAO, which has 20 times more exposure than TA. There are many energy rescaling procedure suggested among experiments (see, e.g., refs. $[16,17,18]$ ) to bring their respective measured fluxes close to each other, mostly at the "ankle" regime. Even these procedures cannot reconcile number of events, after exposure corrections, above $100 \mathrm{EeV}$ among different experiments.

To illustrate the energy rescaling effect on our correlation study, we adopt a recent procedure in ref. [16] which is based on a joint PAO and TA analysis of UHECRs from an overlapping region in the sky. We decrease the energies of the TA events by $25 \%$ but keep the energies of the PAO events unchanged [16]. So the number of UHECRs events above $100 \mathrm{EeV}$ from TA is now 4, out of which only 2 correlates with the neutrino events in the $\delta \chi^{2} \leq 1$ bin (see Fig. 3) in case of first method of analysis. Interestingly, however, there are now 6 counts in the $1 \leq \delta \chi^{2} \leq 2$ bin which corresponds to a Poisson probability of 0.0108 according to the semi-isotropic null. A combined analysis of the TA and PAO data considering the above energy rescaling, gives no significant correlation with neutrino data.

In case of the second method of analysis the maximum relative excess increased to $1.9(1.9 \sigma$ of correlation) in the same bin as earlier, from Figure 4. However for the TA+PAO the maximum relative excess position shifted from $0-0.1 \delta \theta$ to $0.2-0.3 \delta \theta$ bin and the value is now $3.05(3.05 \sigma$ of correlation). 

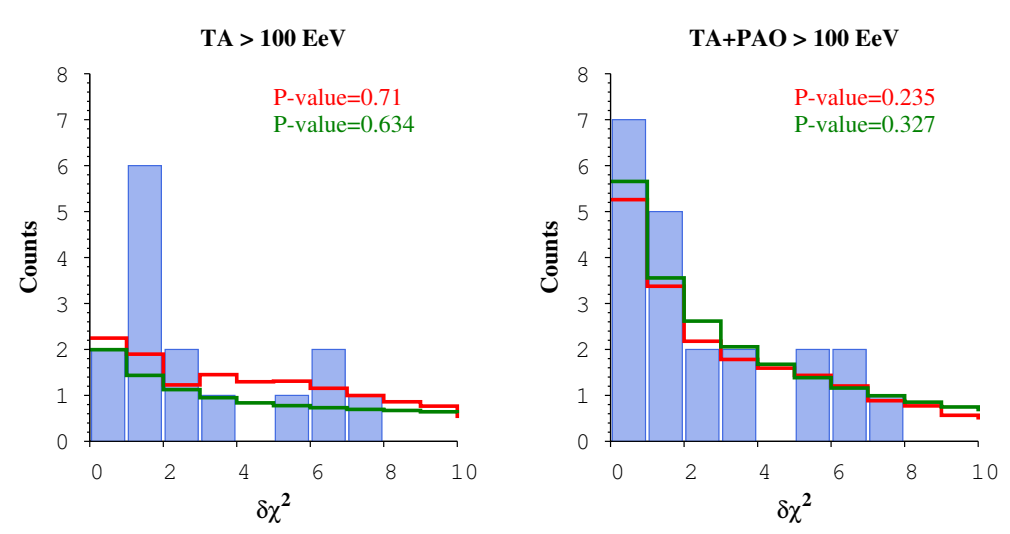

Figure 3: The same as in Figure 1 upper-right and bottom-left panels but the energies for the TA events have been reduced by $25 \%$ compared to the PAO events.
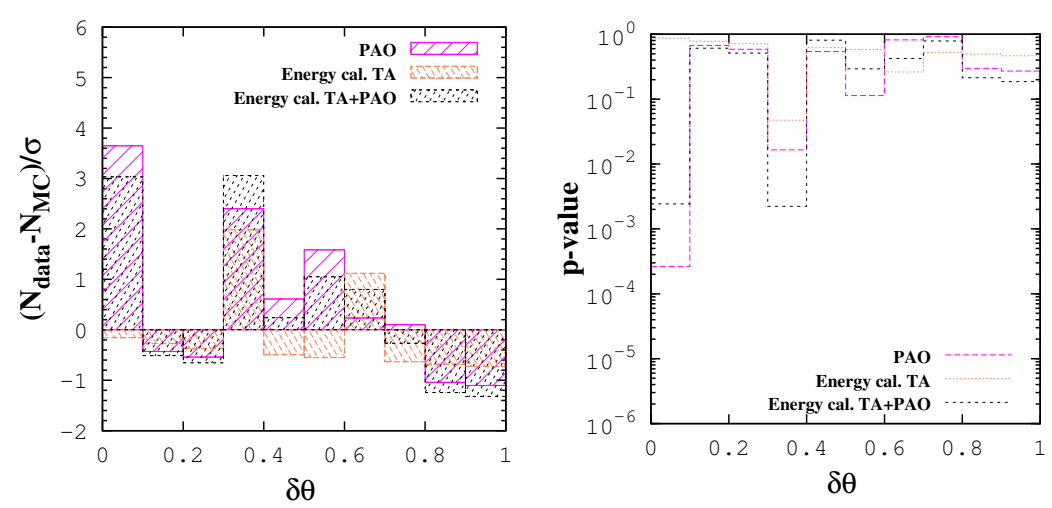

Figure 4: The same as in Figure 2 upper-right and bottom-left panels but the energies for the TA events have been reduced by $25 \%$ compared to the PAO events.

\section{Conclusion}

We have investigated whether the arrival directions of cosmic neutrinos, detected by the IceCube, in first $3 \mathrm{yr}$ and $4 \mathrm{yr}$ are correlated with the arrival directions of UHECRs above energy 100 $\mathrm{EeV}$. In order to test the correlation we have used 2 different methods. The first one is the minimum $\delta \chi^{2}$ invariant statistic method which is constructed from the angle between two unit vectors corresponding to the directions of the neutrino events and UHECRs, and weighted by the angular resolutions of the neutrino events. For this method we have used only the first $3 \mathrm{yr}$ IceCube neutrino events. The second method is Cross correlation. For this analysis we used the $4 \mathrm{yr}$ IceCube neutrino events. We have evaluated the significance of correlation for both the methods by using 
Monte Carlo simulations of randomly generated UHECR directions and comparing with data. We found that IceCube cosmic neutrino events are correlated with the UHECRs with a significance of $90 \% \mathrm{CL}$ in case of first method of analysis. However in the case of second method of analysis the significance increases to more than $3 \sigma$ in the first bin of $\delta \theta$ for PAO only UHECRs, showing a correlation of UHECRs with neutrino events within 1.5 degree (as the average resolution degree of IceCube neutrino events is $15^{\circ}$ ).

\section{References}

[1] M. G. Aartsen et al. [IceCube Collaboration], Phys. Rev. Lett. 113, 101101 (2014) [arXiv:1405.5303].

[2] C. Kopper, ICRC 2015, Netherlands, PoS(ICRC2015)1081

[3] A. Aab et al. [Pierre Auger Collaboration], arXiv:1411.6111.

[4] R. U. Abbasi et al. [Telescope Array Collaboration], Astrophys. J. 790, L21 (2014) [arXiv:1404.5890].

[5] P. Sommers and S. Westerhoff, New J. Phys. 11, 055004 (2009) [arXiv:0802.1267].

[6] K. Greisen, Phys. Rev. Lett. 16, 748 (1966).

[7] G. T. Zatsepin and V. A. Kuzmin, JETP Lett. 4, 78 (1966) [Pisma Zh. Eksp. Teor. Fiz. 4, 114 (1966)].

[8] A. Virmani, S. Bhattacharya, P. Jain, S. Razzaque, J. P. Ralston and D. W. McKay, Astropart. Phys. 17, 489 (2002) [astro-ph/0010235].

[9] P. G. Tinyakov and I. I. Tkachev, JETP Lett. 74, 445 (2001) [Pisma Zh. Eksp. Teor. Fiz. 74, 499 (2001)] [astro-ph/0102476].

[10] C. Bonifazi [Pierre Auger Collaboration], Nucl. Phys. Proc. Suppl. 190, 20 (2009) [arXiv:0901.3138].

[11] M. Takeda, N. Hayashida, K. Honda, N. Inoue, K. Kadota, F. Kakimoto, K. Kamata and S. Kawaguchi et al., Astrophys. J. 522, 225 (1999) [astro-ph/9902239].

[12] M. Nagano and A. A. Watson, Rev. Mod. Phys. 72, 689 (2000).

[13] M. Ave, J. A. Hinton, R. A. Vazquez, A. A. Watson and E. Zas, Phys. Rev. Lett. 85, 2244 (2000) [astro-ph/0007386].

[14] R. U. Abbasi, T. Abu-Zayyad, M. Allen, J. F. Amman, G. Archbold, K. Belov, J. W. Belz and S. Y. BenZvi et al., Astropart. Phys. 30, 175 (2008) [arXiv:0804.0382].

[15] J. Blumer, R. Engel and J. R. Horandel, Prog. Part. Nucl. Phys. 63, 293 (2009) [arXiv:0904.0725].

[16] A. Aab et al. [Telescope Array and Pierre Auger Collaborations], Astrophys. J. 794, no. 2, 172 (2014) [arXiv:1409.3128].

[17] B. R. Dawson et al. [Pierre Auger and Yakutsk and Telescope Array Collaborations], EPJ Web Conf. 53, 01005 (2013) [arXiv:1306.6138].

[18] A. A. Ivanov, Astrophys. J. 712, 746 (2010) [arXiv:1002.2472].

[19] P. Sommers, Astropart. Phys. 14, 271 (2001) [astro-ph/0004016]. 